\title{
Control Line of Sight (LOS) using Robust Control Technique
}

\author{
Deepti Thapliyal ${ }^{1}$ and Deepak Punetha ${ }^{2}$ \\ ${ }^{1}$ Department of Electrical and Electronics Engineering, Tula's Institute, \\ Dehradun, India \\ ${ }^{2}$ Department of Electronics Engineering, Tula's Institute, Dehradun, India \\ er.punetha@gmail.com
}

\begin{abstract}
This manuscript describes the approach to stabilize the line of sight (LOS) of 2-axis gimbal system. For stabilization of LOS the direct approach technique have been used. LOS stabilization is needed in missile guidance, gun target, and handheld cameras. The compensator has been composed utilizing Linear-Quadratic-Gaussian (LQG), LinearQuadratic Regulator (LQR) and H-infinity technique furthermore with PID controller, to meet the prerequisite of following command rate, unsettling influence constriction and stability robustness. The plant model was simulated under disturbance and without disturbance. This paper has also demonstrated bode plot and the step response of $L Q R / L Q G$ and $H$-infinity controller to meet the design specifications. The simulation of the plant model is carried under MATLAB tool. The result collected from $\mathrm{H}$-infinity controller are far better than above all methodologies as it reduces the error up to minimum and also reduces the overshoot in the system and make the system speed faster. It also increases the Gain margin and Phase margin of the system and makes the system without lack of robustness.
\end{abstract}

Keywords: Line of Sight, 2-axis gimbal system, Robustness, LQG/LQR Controller, $H$ infinity technique, Gain Margin, Phase Margin, MATLAB

\section{Introduction}

To control multivariable servo mechanism is difficult task. Cross coupling includes non-linearity that makes the task difficult. These non-linearity and cross coupling disturb the line of sight, so it need to be maintained. LOS stabilization is needed in missile guidance, gun target and handheld cameras. Line of sight control addresses these issues. The LOS slip between a track sensor point and a moving target emerge due toward both focus to-stage kinematics and stage movement disturbances. Most guiding framework, active, when mounted on a versatile stage obliges some manifestation of movement remuneration toward balance out the sensor directing vector along the target line of sight.

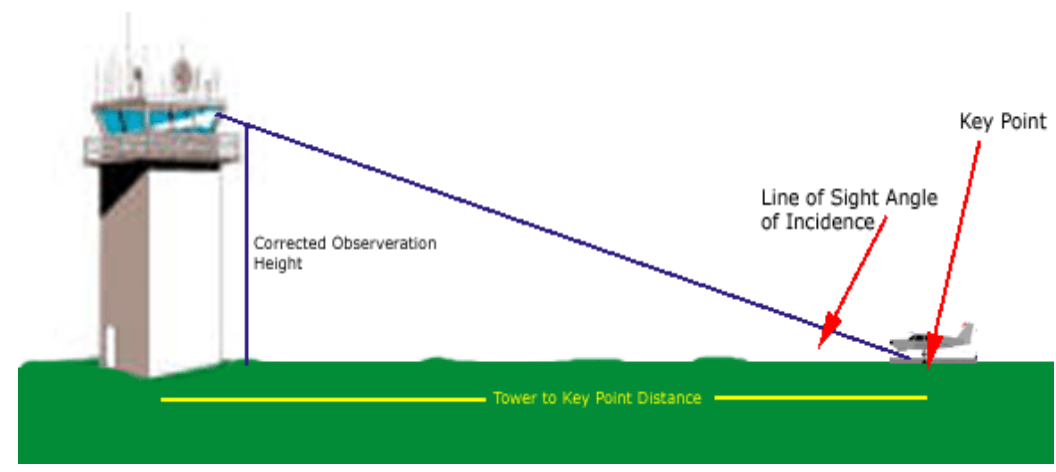

Figure 1. LOS Geometry 
The control issue can be partitioned into two sections: tracking and disturbance rejection. A servo control loop about the track sensor is utilized toward minimized kinematic-induced guiding blunder while inner inertial referenced servo loop gives stage unsettling influence suppression. The technique that addressed the adjustment control issue is frequently referred toward an LOS adjustment; the goal of inertial loop design. LOS is characterized a vector drawn between an observer and the object being observed. The point is the course the eyewitness the really looking or the core of the onlooker's field of view. LOS control addresses the issue of keeping up a sensor's point along the viewable pathway (LOS). Compensating for disturbance to the LOS got from platform pivot or vibration is frequently referred to as LOS adjustment. The major issue can be divided into two loop i.e. tracking and disturbance rejection. Basically, our work or main purpose is to compensate the error or to reduce it to make the Line of sight (LOS) stable so it can point toward the target. To stabilize the line of sight it is important to stabilize vehicle mounted radar and camera used for tracking. The LOS control framework architecture require to attain to an pointing target must be equipped for addressing numerous between many engineering related building issue.

\section{Research Methodology}

The routine configuration methodology is used to build up an adjustment subsystem to minimize inertial jitter and afterward outline a track system. The external effect of environment that disturbs the LOS is compensated in [1]. Two methods were introduced in [2] first the sensor were mounted on gimbal directly to stabilize the mass whose rotation axes define LOS and in second, the rate sensor were mounted to platform measuring platform rate which combines with gimbal angles. Servo drive model for twopivot gimbal adjustment frame to stabilization system and present robust control for air vehicle stabilization system in [3]. Newton first law and second law of motion were introduced in [4] to stabilize and to point the broad array of camera, sensor and military systems. In this approach the outline of an ISP is frequently firmly planned with that of other real sub-frameworks. Keeping up sensor introduction toward an objective is especially testing when the imaging sensor is carried on a versatile vehicle or when the objective is exceedingly dynamics, [5] describes the design of ISP's and target tracker for imaging optical objects. In [6] the key believe is to upgrade the instinctive decoupled controller structure with estimation of camera inertial angular rate and its optical turn. Arrangement uses extra information in [7] from an inertial exact rate sensor; specifically, the exact rate of the payload (camera, laser) around its optical axis. This paper shows a control application for the inertial adjustment of a gyroscopic stage with two degrees of opportunity (2-DOF). The reasons for this application are, in the first place, to control the precise positions of the stage without inertial unsettling influences and second, to control speeds measured in an inertial casing, while dismissing the disturbances connected with moving parts [8].

In the proposed work an optimal solution of the LOS stabilization problem, through the systematic application of the linear quadratic Gaussian (LQG) theory and LQR in order to stabilize the rate of the line of sight, meeting the stringent requirements of fast tracking, disturbance attenuation and robust stability. The PID controller is implemented to compensate the random error introduced in the system. The proposed work demonstrated the bode plot the step response of LQR/LQG and H-infinity controller to meet the design specifications.

\section{Design and Control of LOS Stabilization System}

Various issues experienced by an analyst or expert are multidisciplinary in nature. Without related information, a proper arrangement obviously of activity may not be clear. The sensor mounted on multi-pivot gimbal, is secured to the subject of the vehicle [9]. 


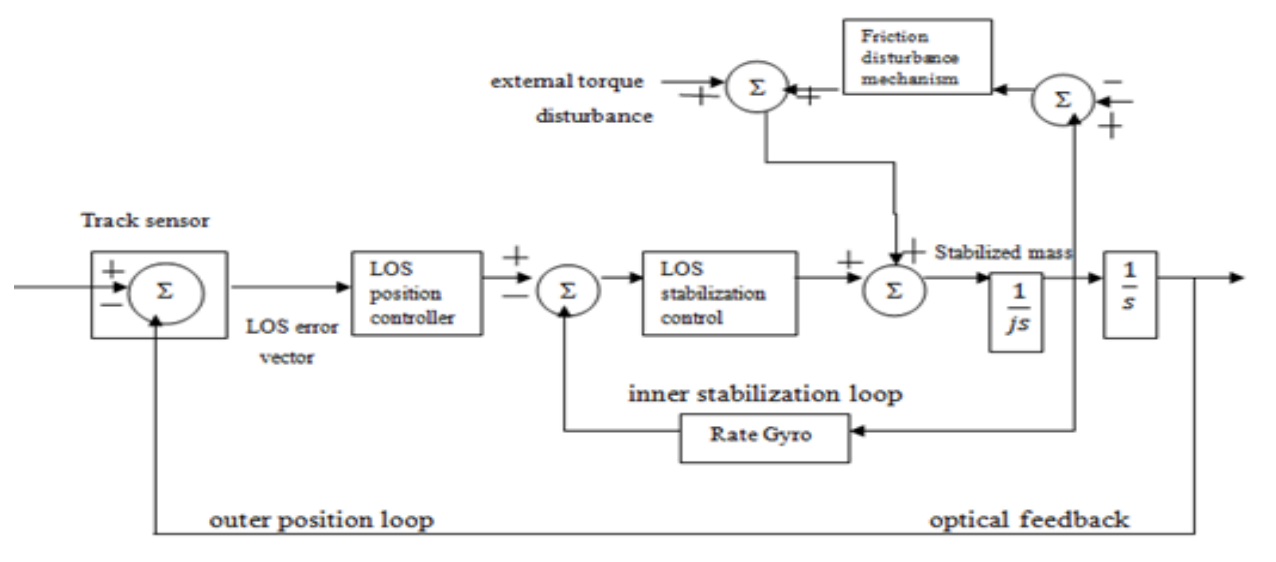

Figure 2. LOS Pointing Control Architecture

There are two servo control circle, an internal LOS adjustment control circle, and an external LOS position circle. The external circle sensor is a camera or electro-optical following sensor. This control design is for engine driven position servo internal rate criticism. The inward circle is then inertial rate circle whose goal is to disconnect aggravation from external position circle, minimizing the inclination blunder in the plane orthogonal to the LOS vector because of torques (i.e. contact, imbalance), platform movement and commotion. Aggravation weakening results by utilizing high pick up pay as a part of the adjustment control circle [10].

\subsection{Direct LOS Stabilization}

There are a few sort of inertial rate detecting gadgets that can be utilized with rate and rate incorporating gyros. The two most common adjustment control framework structures are: Direct and Indirect LOS Stabilization. Direct LOS conformity is usually endorsed for exactness coordinating applications. The inertial rate sensors are incorporated with the body toward be offset. The course packaging joined to the offset body is the 'internal turn pivot or sensor bearing blueprint whose $\mathrm{X}$-hub describes the controlling vector. This setup particularly resources precise rates orthogonal toward the LOS vector, to inside the track mistake [11-12].

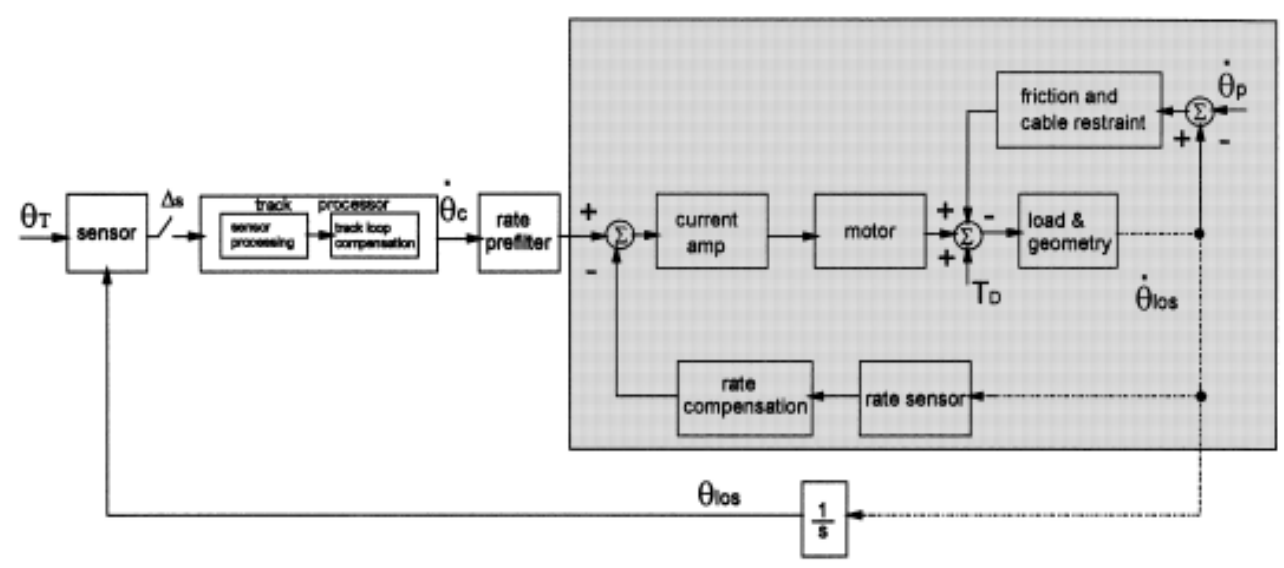

Figure 3. Direct LOS Stabilization 


\subsection{Indirect LOS Stabilization}

With Indirect LOS modification the rate sensors are mounted on the gimbal outside pivot to sense stage development. This system is now and again alluded to as a strapdown setup, as the inertial rate sensors are strapped down to the gimbal base or stage. It can fulfill a bit of the issues associated with the immediate adjustment arrangement, especially estimate and obliging high slew rates. Three rate sensors are obliged to correctly quantify the stage rate.

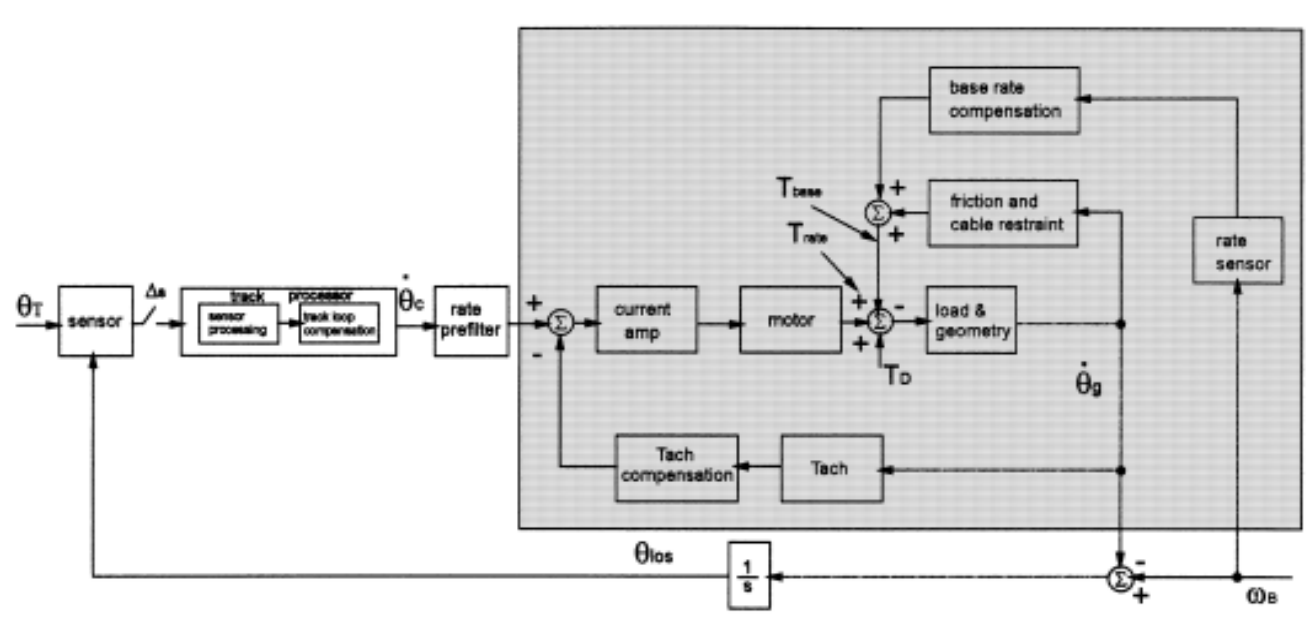

Figure 4. Indirect LOS Stabilization

\section{Modeling of LOS Stabilization and Subsystem}

The plant under study is dissected in point of interest. It expresses the run of the mill outline prerequisite of the mechanical part of the plant [13-14].

\subsection{Mechanical Parameters Affecting the Design}

Friction is an essential driver for the unsettling influence transmission to the balanced out component (LOS). This can be grouped into three classifications viz. sitction, Coulomb friction and the viscous friction. The backlash is normally present in a given system. This results into the introduction of the dead band. Resonance occurs because of the various coupling factors between the actuator and the load.

\subsection{Components of the Plant}

Figure shows the block diagram for the plant dynamics. It indicates the transfer functions of the various blocks constituting the plant. 


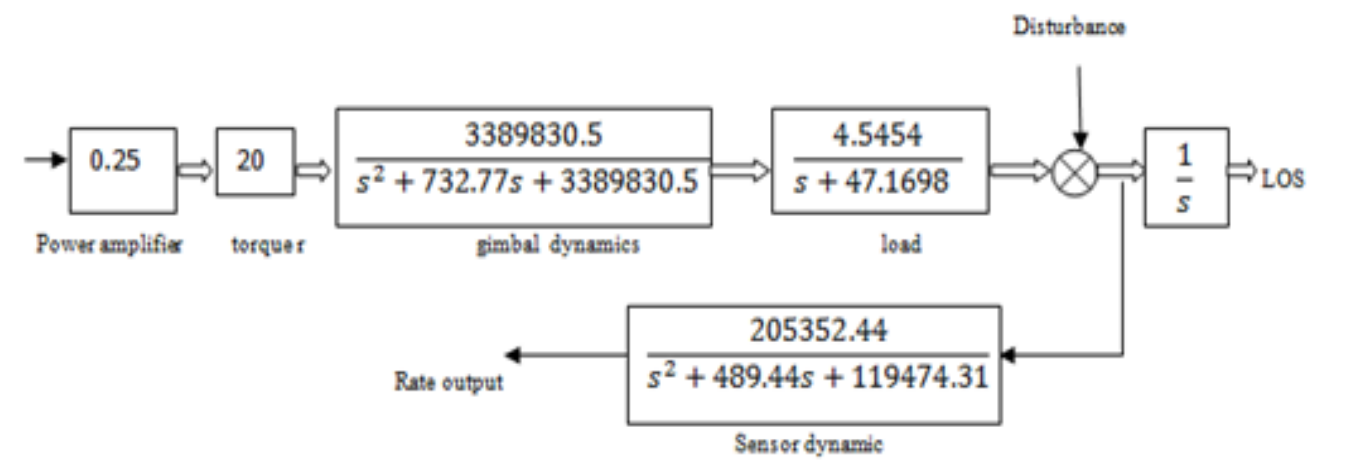

Figure 5. Block Diagram of the Plant Dynamics

\subsection{State Space Representation of the Plant}

The dynamics of the system can be expressed by the following differential equations:

$$
\dot{\mathrm{G}} \mathrm{o}(\mathrm{t})=-489.44 \mathrm{G}^{\circ} \mathrm{o}(\mathrm{t})-119474.31 \mathrm{Go}(\mathrm{t})+205352.44 \mathrm{Ro}(\mathrm{t})
$$

Or,

$$
\mathrm{Go}(\mathrm{t})=-489.44 \mathrm{Go}(\mathrm{t})-119474.31 \mathrm{Go}(\mathrm{t})+205352.44 \mathrm{R}(\mathrm{t})+205352.44 \mathrm{~d}(\mathrm{t})
$$

Since, $\operatorname{Ro}(\mathrm{t})=\mathrm{R}(\mathrm{t})+\mathrm{d}(\mathrm{t})$

and,

$$
\mathrm{T}(\mathrm{t})=-732.77 \mathrm{~T}(\mathrm{t})-3389830.5 \mathrm{~T}(\mathrm{t})+35593220.25 \mathrm{u}(\mathrm{t})
$$

Therefore, the state space representation of the plant with usual matrices $\mathrm{A}, \mathrm{B}, \mathrm{C}, \mathrm{D}$, and $\mathrm{E}$ as:

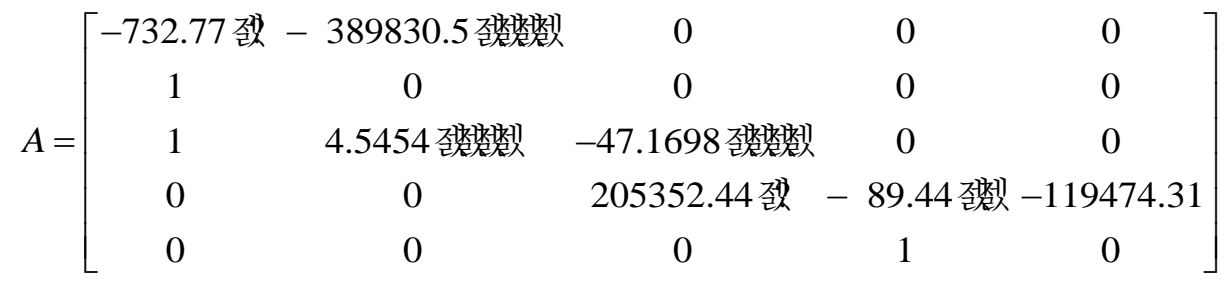

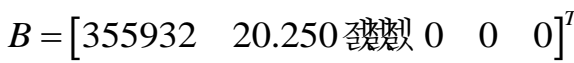

$C=\left[\begin{array}{lllll}0 & 0 & 0 & 0 & 1\end{array}\right]$

$D=[0]$

$E=\left[\begin{array}{lllll}0 & 0 & 0 & ? 0532.44 & 0\end{array}\right]^{T}$

$F=[0]$

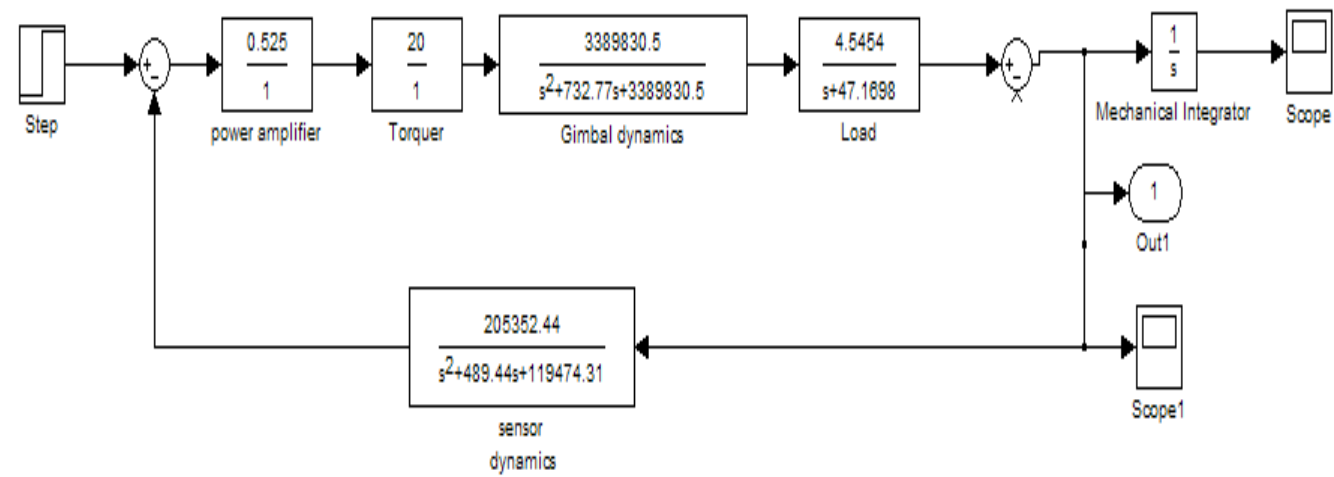

(a) Model of Plant under No Disturbance 


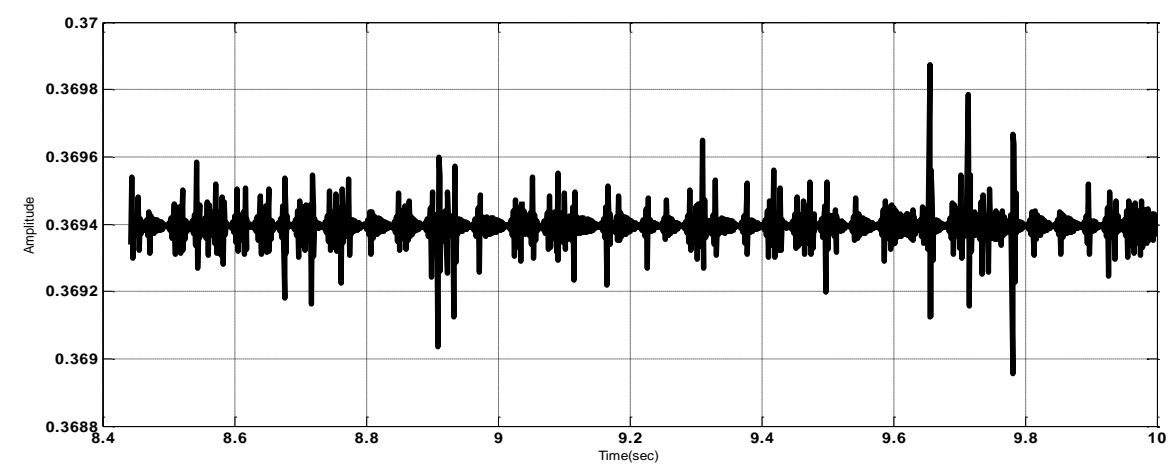

(b) Rate Output (Time/Amplitude) Response

Figure 6. (a) The Parameter of the Plant has bezzzzzzz en Already Discussed so Using that Parameters the Block Diagram of Plant is Designed. The Response of the Plant is First Examined without Using PID Controller under No Disturbance. (b) Depicts that Rate Output and Its Standard Deviation is 8.281 Micronsh

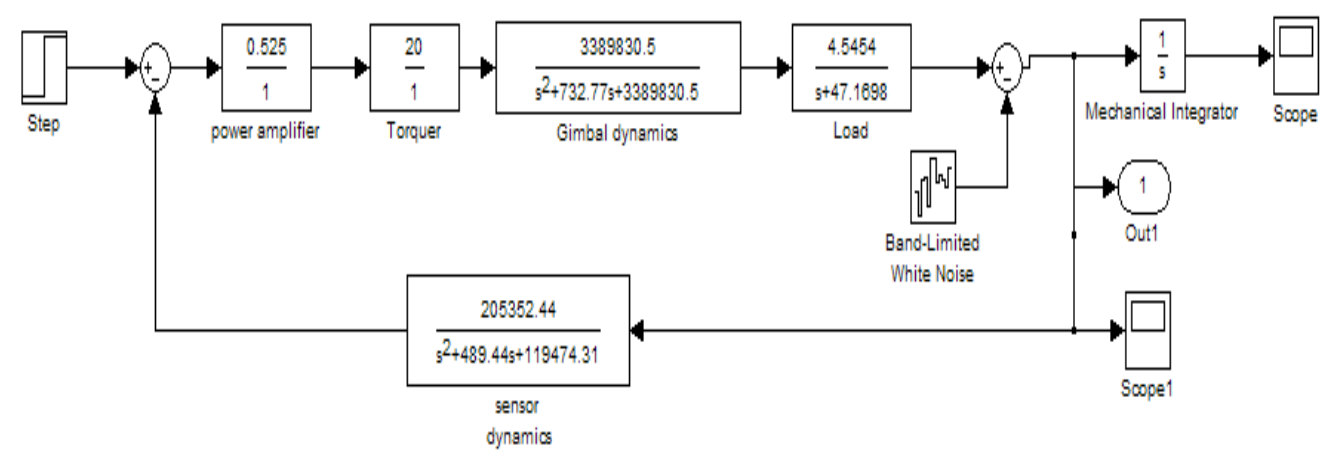

(a)

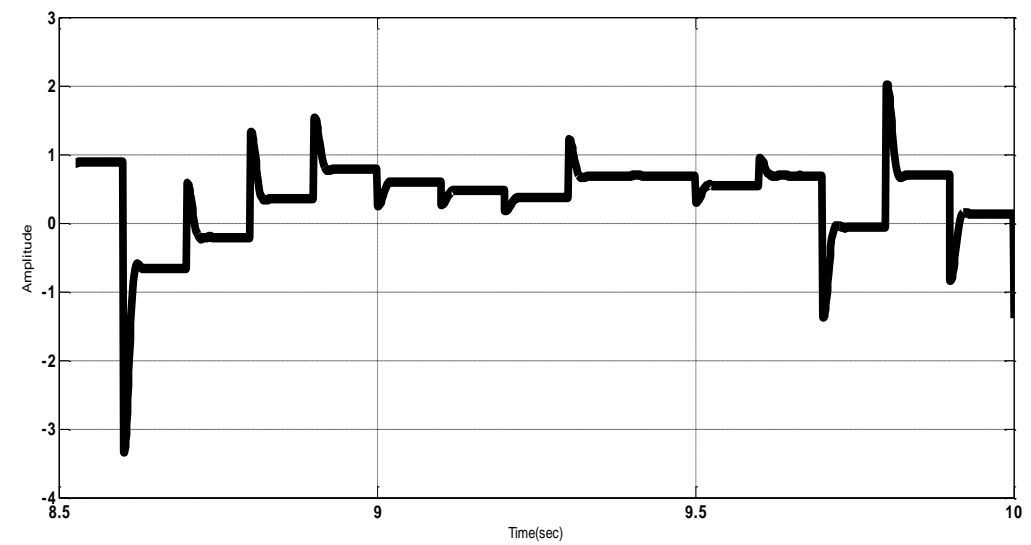

(b)

Figure 7. (a) Model of Plant in Presence of External Disturbance, (b) Rate Output Under External Disturbance

Figure 7. (a) shows the plant under random noise i.e. Band-Limited white noise, Figure shows the response of the model that depicts the nature of the disturbance. The noise power of this type of disturbance is taken as 0.1 and its standard deviation is evaluated as 0.4293 . 


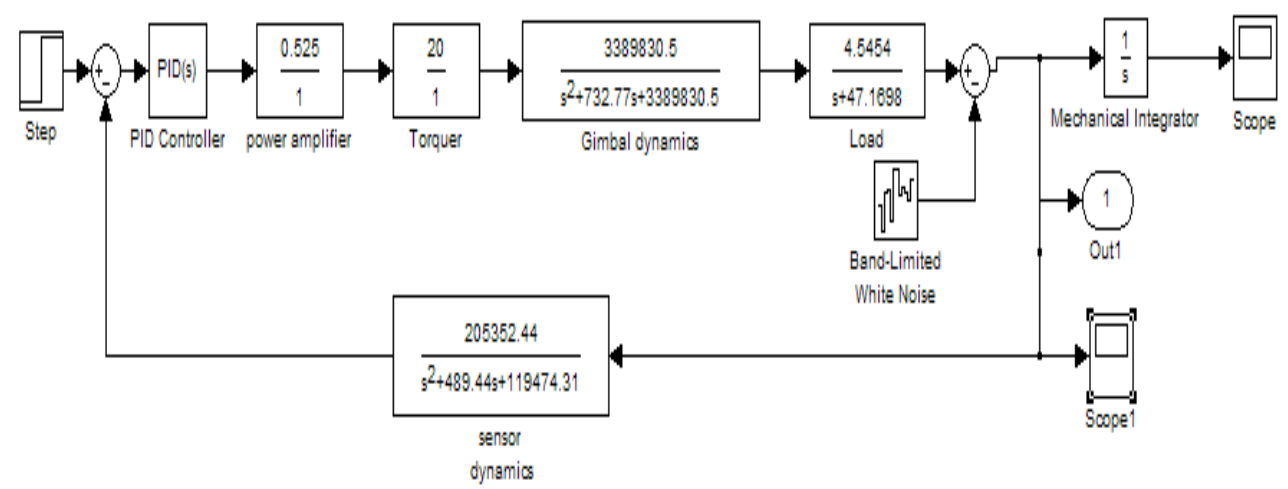

(a)

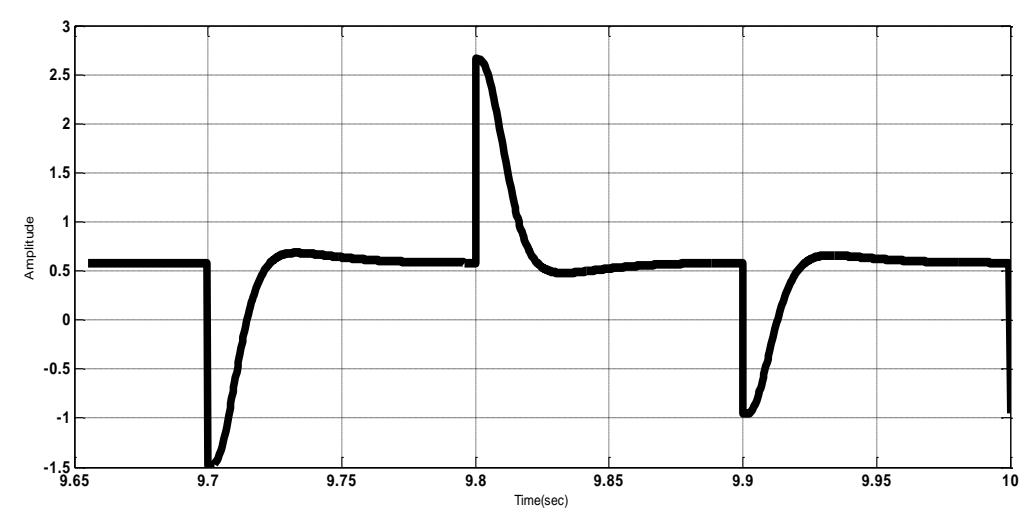

(b)

Figure 8. (a) Plant Model with PID Controller, (b) Rate Output Examined under PID Controller

Figure 8. (a) The model is examined under PID controller having the noise power of 0.1. In Figure the noise or the disturbance is somewhat affected by PID controller as its standard deviation is calculated as 0.9997. In the following table standard deviation is calculated for different value of noise power.

Table 1

\begin{tabular}{|c|c|c|}
\hline Parameters & Noise power & Standard deviation \\
\hline $\begin{array}{c}\text { Without PID } \\
\text { controller }\end{array}$ & 0.1 & 0.4293 \\
\hline $\begin{array}{c}\text { With PID } \\
\text { controller }\end{array}$ & 0.1 & 0.0997 \\
\hline
\end{tabular}

Hence, the table shows the value of standard deviation decreases for the same value of noise power in the presence of PID controller. 


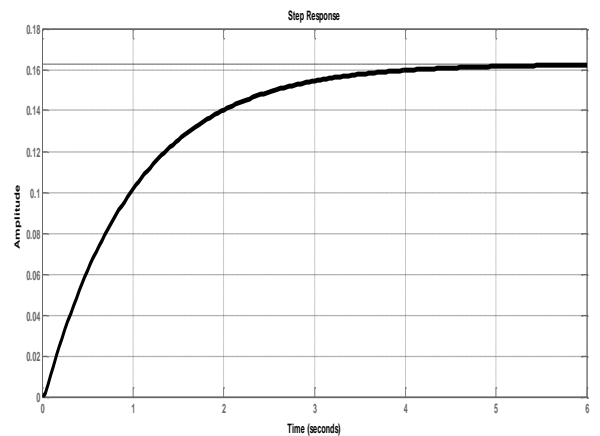

(a)

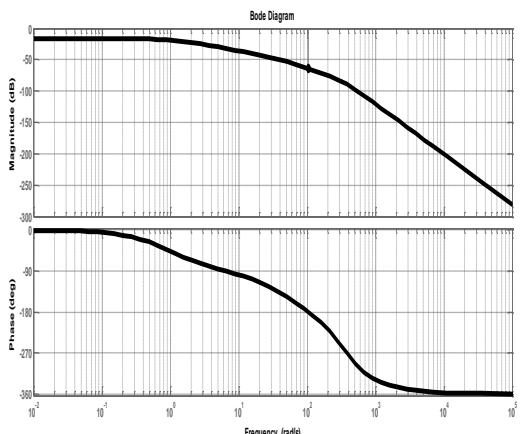

(b)

Figure 9. (a) Step Response of the Plant Using LQR Controller (b) Bode Plot of the Plant Using LOR Controller

Figure 9. (a) The step response of the plant with LQR controller is shown in Figure 6.7 using the transfer function without adding disturbance which shows the Rise time=2.184 $\mathrm{sec}$, settling time $=3.9225 \mathrm{sec}$ and overshoot $=0 \%$. The response of $\mathrm{LQR}$ controller is having more rise time and settling time which shows that system is tending towards stability after so much time. Hence it is concluded that the system is stable but it is taking more time to reach stability so further controller are used.

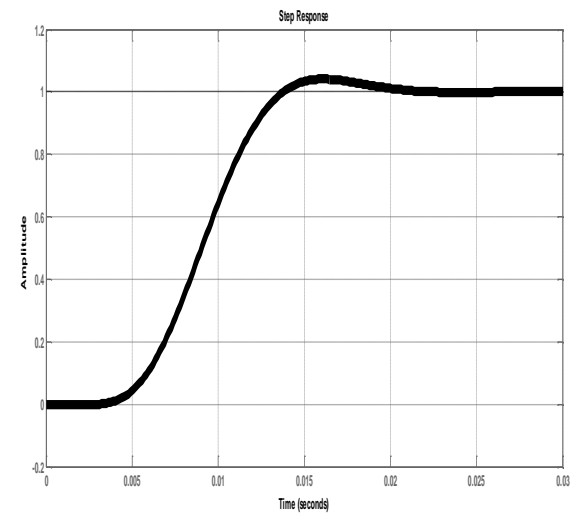

(a)

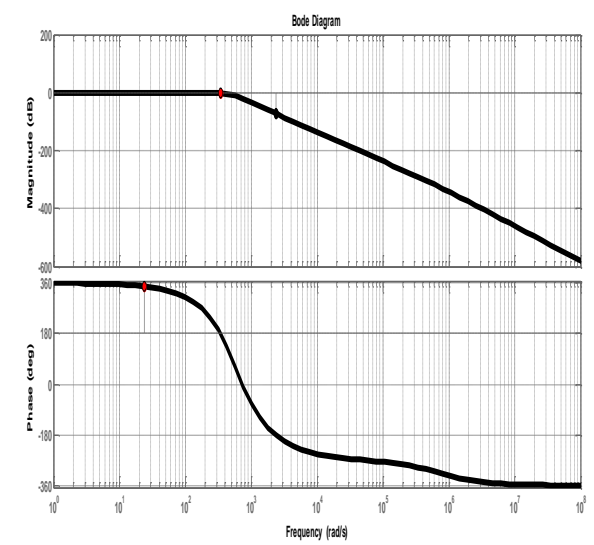

(b)

Figure 10. (a) Step Response of the Plant Under LQG Controller, (b) Bode Plot of the Plant Under LQG Controller

The step response of the plant with LQG controller is shown in Figure 10. (a) Which shows the Rise time $=0.063 \mathrm{sec}$, settling time $=0.0188 \mathrm{sec}$ and overshoot $=3.9956 \%$ and the bode plot response of the plant is shown in Figure which shows the G.M=2.53 dB and phase margin=168 degree. The settling time is less as compare to the LQR controller. Hence shows the stability of the system. 


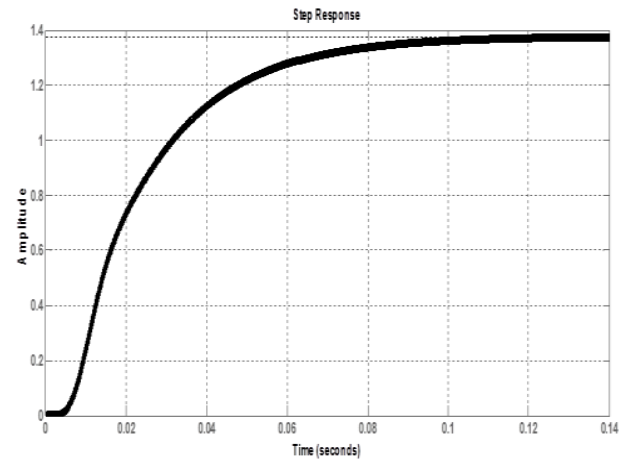

(a)

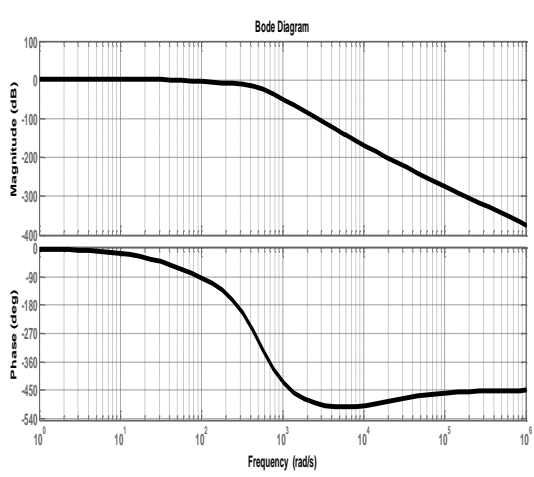

(b)

Figure 11. (a) Step Response Under H-Infinity Controller, (b) Bode Plot Under H-Infinity Controller

In Figure 11. (a) step response of the plant using H-infinity controller shows Rise time $=0.0442$, Settling Time $=0.0864$ and overshoot $=0 \%$ and the bode plot in Figure shows Gain margin $=10.8 \mathrm{~dB}$ and Phase margin $=132 \mathrm{deg}$. Here the rise time and settling time of the response is far much better than PID controller and LQG/LQR controller which conclude that the system is stable and is having less settling time as compare to other controllers.

Table 2

\begin{tabular}{|c|c|c|c|c|}
\hline $\begin{array}{c}\text { Performances } \\
\text { Indices }\end{array}$ & $\begin{array}{c}\text { PID } \\
\text { controller }\end{array}$ & $\begin{array}{c}\text { LQR } \\
\text { controller }\end{array}$ & $\begin{array}{c}\text { LQG } \\
\text { controller }\end{array}$ & $\begin{array}{c}\text { H-infinity } \\
\text { controller }\end{array}$ \\
\hline Rise time (sec) & 0.0135 & 2.18733 & 0.0146 & 0.0442 \\
\hline Settling time(sec) & 0.0558 & 3.9196 & 0.0193 & 0.0864 \\
\hline Overshoot (\%) & 4.97 & 0 & 2.1251 & 0 \\
\hline Gain margin(dB) & 28.2 & 60.6 & 2.53 & 10.8 \\
\hline Phase margin (deg) & 120 & Infinity & 168 & 132 \\
\hline
\end{tabular}

\section{Conclusions}

The LQR/LQG methodology was implemented for the given plant. All the design specification was met. This method gives an excellent way of posing all the requirements in the frequency domain. Further the plant model was simulated under disturbance and without disturbance. The PID controller compensates the error to minimum. With the PID controller the standard deviation has been reduced up to 0.0997. Above all methodologies used it is concluded that $\mathrm{H}$-infinity gives the best response than all other controller used. It reduces the error up to minimum and also reduces the overshoot in the system and makes the system speed faster. The value of Rise time and settling time evaluated using $\mathrm{H}$-infinity controller is 0.0442 and 0.0864 . It also increases the Gain margin and Phase margin of the system and makes the system without lack of robustness.

\section{References}

[1] M.K. Masten, H.R. Sebesta, "Line-of-Sight Stabilization/Tracking Systems: An Overview" InAmerican Control Conference, IEEE, (1987), pp. 1477-1482.

[2] P.J. Kennedy, R.L Kennedy, "Direct versus indirect line of sight (LOS) stabilization", IEEE Transactions on control systems technology, vol.11, no. 1, (2003), pp. 3-15.

[3] H.P. Lee, I.E. Yoo, "Robust control design for a two-axis gimbaled stabilization system", InAerospace Conference, 2008 IEEE, (2008), pp. 1-7. 
[4] J.M. Hilkert, "Inertially stabilized platform technology concepts and principles", IEEE Control Systems. vol. 28 , no. 1, (2008), pp. 26-46.

[5] M.K. Masten, "Inertially stabilized platforms for optical imaging systems", IEEE Control Systems, vol. 28, no. 1, (2008), pp. 47-64.

[6] Z. Hurak, M. Rezac, "Image-based pointing and tracking for inertially stabilized airborne camera platform”, IEEE Transactions on Control Systems Technology, vol. 20, no. 5, (2012), pp.1146-59.

[7] F.R. Rubio, M.G. Ortega, F. Gordillo, M. Vargas, "Application of position and inertial-rate control to a 2-DOF gyroscopic platform”, Robotics and Computer-Integrated Manufacturing, vol. 26, no. 4, (2010), pp. 344-53.

[8] N.H. Mackworth, "A stand camera for line-of-sight recording”, Perception \& Psychophysics, vol. 2, no. 3, (1967), pp. 119-27.

[9] A. Rue, "Stabilization of precision electrooptical pointing and tracking systems", IEEE Transactions on Aerospace and Electronic Systems. 5(AES-5), (1969), pp. 805-19.

[10] Z. Hurak, M. Rezac, "Image-based pointing and tracking for inertially stabilized airborne camera platform", IEEE Transactions on Control Systems Technology, vol. 20, no. 5, (2012), pp. 1146-59.

[11] C. Fu, W. Ma, "Partial stability of some guidance dynamic systems with delayed line-of-sight angular rate", International Journal of Control, Automation and Systems, vol.12, no. 6, (2014), pp.1234-44.

[12] B.J. Murphy, G.M. Haddad, R.M. Stelnman, "Simple forms and fluctuations of the line of sight: Implications for motor theories of form processing", Perception \& Psychophysics, vol. 16, no. 3, (1974), pp.557-63.

[13] J. Sostrin, "Determine Your Line of Sight", InThe Manager's Dilemma, Palgrave Macmillan US, (2015), pp. 55-67.

[14] Z. Lin, K. Liu, L. Zhang, D. Zeng, "Coupling effect and control strategies of the maglev dual-stage inertially stabilization system based on frequency-domain analysis", ISA transactions, (2016). 